\title{
Regard sur le développement régional Quel passé... quel avenir pour les régions nordiques du Québec
}

\author{
Camil Girard ${ }^{1}$ et Laval Gagnon ${ }^{2}$
}

\section{La réingénérie de l'État et ses impacts sur les régions nordiques}

Quel avenir y a-t-il pour les régions nordiques dans les nouveaux projets de "réingénérie » ou de «modernisation » de l'État? Selon les propos tenus par le Premier ministre du Québec, Jean Charest, la modernisation de l'État québécois aurait commencé en 1960. Cela peut se vérifier assez facilement lorsqu'il s'agit de montrer que, depuis les années 1960, les régions du Québec se sont développées dans un contexte où l'État, et en particulier l'État provincial, a défini, par son mode de gestion et d'intervention de territoires plus nordiques et marginaux, son rôle en tant que gouvernement. Ce faisant, le Québec a montré, par ses actions en région, que l'intégrité de son territoire passait par une occupation effective et une mise en valeur de l'ensemble du territoire.

Nous posons ici comme hypothèse que le rôle des gouvernements au Québec, et en particulier celui du Québec, s'est défini au $\mathrm{Xx}^{\mathrm{e}}$ siècle dans un contexte particulier qui s'inscrit en continuité avec une gestion de territoires nordiques que l'on qualifie de périphériques.

Le cas du Saguenay-Lac-Saint-Jean sert ici de cadre plus particulier mais non limitatif pour illustrer comment, à travers les politiques gouvernementales appliquées à la marge territoriale, le Québec en vient à définir son rôle en région. La seconde partie du texte décrit à grands traits l'intervention régionale de l'État à la fin des années 1960 et dans les années 1970, et son désengagement graduel à compter des années 1980. Nous proposons finalement quelques éléments de prospective territoriale qui permettraient de dégager une vision articulée et documentée de l'avenir du Saguenay-Lac-Saint-Jean et de favoriser une démarche de prise en charge des populations ( $\mathrm{y}$ compris les nations autochtones) avec la complicité et le support des corporations, des élus et de l'État.

\section{Quel rôle pour l'État en périphérie ?}

Dans le débat actuel sur la place de l'État dans le développement, il ne s'agit pas de prétendre qu'il faut plus ou moins d'intervention de l'État en région ou si la place du privé est souhaitée. Il s'agit plutôt de découvrir et de mieux comprendre comment les États ont géré le territoire à la périphérie des centres plus populeux en s'appuyant de manière systématique sur l'entreprise privée. En développant des modes d'interventions complexes et multiples qui laissent une place prépondérante aux entreprises privées, les rapports du capital et de l'État se sont définis au $\mathrm{XX}^{\mathrm{e}}$ siècle autour de conceptions qui empruntent au mode d'État industriel, lequel tend à créer des conditions favorables au développement afin de laisser aux grandes entreprises la responsabilité de la mise en valeur des territoires à partir des ressources naturelles.

La réingénérie ou la modernisation qui est défendue de nos jours prétend que le capital et le marché doivent désormais être laissés à eux-mêmes, alors que l'État interviendrait moins. Dans ce contexte, les régions se sentent piégées par un discours qui tend à les associer à des espaces marginaux, éloignés, coûteux et peu peuplées. En contrepartie, il existe aussi un discours régional qui veut que les régions soient pillées de leurs ressources par des capitalistes et des gouvernements qui ne desservent que leurs actionnaires ou les populations des grands centres. Les per- 
tes d'emplois dans les secteurs de l'aluminium et des pâtes et papier, avec les conséquences que cela a sur la décroissance de la population et sur les secteurs plus névralgiques de l'éducation et de la santé, contribuent à entretenir de part et d'autre ces mythes sur les régions.

Il faut tout de même rappeler l'importance des ressources naturelles dans l'économie des régions et du Québec actuel. En 2000, le produit intérieur brut (PIB) du secteur des ressources naturelles se situait, selon l'Institut de la statistique du Québec, à 23 milliards de dollars (PIB global au Québec 230 milliards\$) avec la création de près de 300000 emplois directs et indirects. En 2001, la valeur des exportations des ressources naturelles atteint aussi 23,3 milliards\$ (34\% des exportations du Québec dans la catégorie). Le PIB du secteur énergétique s'établissait à lui seul à 8,2 milliards $\$$ en 2000 . Le Nord-du-Québec héberge $49 \%$ de la production installée d'Hydro-Québec, et la Côte-Nord, 26,8 \% (total $75,8 \%$ ). Hydro fait travailler $9,6 \%$ de ses effectifs (près de 2000 emplois) sur ce territoire. Le ministère des Ressources naturelles, à titre d'exemple, procure 108 emplois sur la Côte-Nord et 46 dans le Nord-duQuébec (4\% sur un total de 3517 emplois). Dans le secteur privé, généralement considéré comme la contrepartie de l'exploitation des ressources naturelles par la grande entreprise, les emplois ont connu un déclin graduel, alors que la productivité globale n'a cessé de croître avec les changements technologiques et les politiques de rationalisation.

Bref, l'exploitation des ressources naturelles a connu une croissance continue en termes de productivité et de valeur, et une décroissance constante en retombées sur le territoire, particulièrement dans le domaine de l'emploi, en particulier pour les jeunes. Un contrat de plus en plus inégal. Donc, si les mythes restent entretenus par le centre à l'effet que les régions ressources sont les parents pauvres de l'économie du Québec, les régionaux se sentent flouées par ce discours.

\section{Le territoire québécois... un pays renversé}

Le Québec est un pays renversé. Quelle place occupe le territoire plus nordique (86\%) qui reste ultimement immense, peu peuplé, isolé, fragilisé dans un Québec où la population se concentre près de sa frontière sud ? L'occupation de ces territoires nordiques reste limitée par une multitude de contraintes dont le climat et l'immensité du territoire sont les plus importants. Revoir notre histoire, c'est découvrir un Québec central qui reste frileux à reconnaître le nord comme partie intégrante du territoire du Québec.

Est-il besoin de rappeler que $92 \%$ de tout le territoire québécois est constitué de terres publiques? Le centre (le sud) du Québec comprend $15 \%$ du territoire et regroupe $88 \%$ de la population du Québec. Les régions périphériques regroupent 548000 personnes, soit $7,5 \%$ de la population sur le versant nord du fleuve dans les régions de l'Abitibi, du SaguenayLac-Saint-Jean et de la Côte-Nord. Ce territoire est presque aussi grand que la France avec ses 467000 kilomètres carrés (31\% du territoire québécois) et est encore essentiellement géré en des terres publiques mises en valeur par de grandes entreprises forestières ou hydro-électriques. Enfin, 55 \% du territoire québécois est compris dans les territoires des Cris et des Inuits (Nord-du-Québec) avec ses $840000 \mathrm{~km} 2$ et ses 39000 habitants.

\section{L'exploitation des ressources naturelles a connu une croissance continue en termes de productivité et de valeur, et une décroissance constante en retombées sur le territoire, particulièrement dans le domaine de l'emploi, en particulier pour les jeunes. Un contrat de plus en plus inégal.}

\section{Un Moyen-Nord... isolé et dispersé}

Le peuplement principal des trois régions du moyennord se situe dans quelques agglomérations urbaines (Rouyn-Noranda, Baie-Comeau, Sept-Îles, Saguenay). Pour le reste, les populations de ces territoires sont dispersées et elles restent isolées et mal intégrées au territoire québécois. Sous ce rapport, Saguenay serait l'exemple d'une ville nordique marquée à la fois par une certaine intégration à l'économie centrale et internationale tout en agissant comme pôle d'attraction et de développement de son arrière-pays nordique. À l'autre extrême de la faible intégration à l'économie, nous retrouvons, sur la grande majorité des territoires, les peuples autochtones qui cherchent à se prendre en charge à partir de leurs territoires ancestraux. La Convention de la Baie James, la Paix des Braves et l'Approche commune s'inscrivent dans ce mouve- 
ment de reconnaissance de droits historiques des peuples autochtones sur un territoire où la Couronne a toujours la souveraineté territoriale avec les peuples autochtones.

Autre réalité souvent oubliée, ce sont les populations non autochtones isolées qui, comme sur la BasseCôte-Nord, ne sont toujours pas reliées au Québec par voie routière en 2004. Seuls des projets de mise en valeur de ressources, de barrages hydro-électriques et de coupe forestière permettraient de développer cette dernière frontière du nord-est québécois.

On le voit ici, les populations du moyen-nord restent concentrées dans quelques zones urbaines ou dispersées et isolées sur des territoires municipalisés qui apparaissent minuscules lorsque comparés aux territoires publics.

Une relecture des tendances lourdes de l'action des gouvernements provinciaux au $\mathrm{XX}^{\mathrm{e}}$ siècle $\mathrm{s}$ 'inscrit donc dans ce contexte où l'élargissement des frontières de la province appelle une organisation particulière des territoires marginaux. Dans ce contexte, les gouvernements sont confrontés à assurer le développement de l'ensemble du territoire afin d'en assurer l'intégrité.

\section{Les populations du moyen-nord restent concentrées dans quelques zones urbaines ou dispersées et isolées sur des territoires municipalisés qui apparaissent minuscules lorsque comparés aux territoires publics.}

Dans la gestion des territoires nordiques, au-delà des territoires municipalisés, seuls les peuples autochtones ont occupé effectivement les territoires nordiques jusqu'en 1840 avec les compagnies qui exploitaient les ressources à partir des territoires ancestraux (fourrure, bois, eau). Les États n'ont jamais affirmé leur intégrité dans les territoires nordiques. Sous ce rapport, la perte du territoire du Labrador en 1927 montre que le Québec n'a pas pu confirmer devant les tribunaux l'intégrité de ce territoire parce qu'il ne l'avait pas développé. Il n'a pu montrer que des alliances avec les nations autochtones du Labrador avaient existé. La Convention de la Baie-James et du Nord québécois (1975) ainsi que l'Approche com- mune (2004) viennent confirmer que le Canada, le Québec et les nations autochtones partagent un territoire dont l'intégrité n'est assurée que parce que des populations et leurs gouvernements l'occupent et le mettent en valeur depuis des temps immémoriaux et que cela devra continuer en s'adaptant aux réalités contemporaines.

\section{Histoire d'une mort annoncée et d'une renaissance attendue}

Stimulé et nourri par la montée et l'affirmation du mouvement nationaliste qui s'est cristallisé par la Révolution tranquille, l'État québécois a jeté les bases et articulé, dans les années 1960 et 1970, un modèle social construit autour d'une philosophie gouvernementale largement interventionniste et ouverte à une participation des citoyens à la gestion des services sur le territoire, notamment en affaires sociales, en santé, en éducation, en culture et en développement régional.

Financé par une politique budgétaire expansionniste, l'État a mis en place des institutions, des organismes et des programmes d'inspiration social-démocrate qui ont été appliqués dans le Québec municipalisé, notamment à travers l'approche des régions administratives.

Ainsi le Saguenay-Lac-Saint-Jean, comme les autres régions du Québec, a connu dans les années 1970 une période extrêmement fertile en implantation d'infrastructures qui a mobilisé d'importants capitaux publics : le réseau de l'Université du Québec, le réseau des cégeps, la construction de polyvalentes, la mise en place du réseau de la santé et des services sociaux, la présence d'Hydro-Québec dans les régions et dans le moyen-nord, particulièrement par la construction des grands barrages, la présence croissante et l'allocation plus ou moins importante de ressources de divers ministères dans plusieurs régions (forêts, chasse et pêche, tourisme et loisirs, culture, travaux publics, transports, infrastructures municipales, etc.).

Quoique moins sentie, l'action du gouvernement fédéral, au Saguenay-Lac-Saint-Jean comme ailleurs, a été néanmoins tangible : centre des données fiscales, aide à l'agriculture, bureaux d'emploi et d'assurancechômage, base militaire, transport aérien et ferroviaire, présence influente du défunt ministère de l'Expan- 
sion économique régionale, installations portuaires, etc.

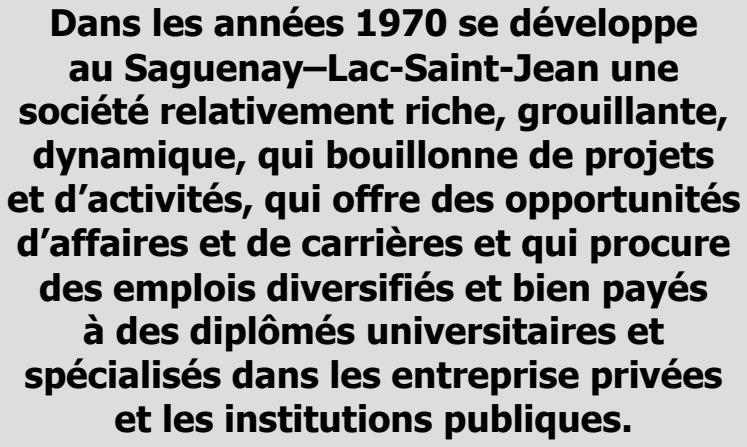

Dans les années 1970 se développe au Saguenay-Lac-Saint-Jean une société relativement riche, grouillante, dynamique, qui bouillonne de projets et d'activités, qui offre des opportunités d'affaires et de carrières et qui procure des emplois diversifiés et bien payés à des diplômés universitaires et spécialisés dans les entreprise privées et les institutions publiques.

L'interventionnisme de l'État a intensifié l'élan socioéconomique des territoires régionaux, dont le Saguenay-Lac-Saint-Jean qui a connu une période d'effervescence avec la multiplication des médias privés (journaux régionaux, stations de radio et de télévision), des services spécialisés et professionnels du secteur tertiaire souvent en lien étroit avec la grande entreprise comme Alcan, Abitibi-Consol, HydroQuébec (bureaux d'avocats, firmes d'ingénieurs, architectes, courtiers en valeurs mobilières, etc.), la construction de centres commerciaux, d'édifices (publics et privés), de routes, etc.

Finalement, un mouvement de prise en charge économique par la gestion et l'exploitation du capital local émerge à travers la croissance du Mouvement Desjardins et de la Fédération des caisses d'entraide économique en région.

Ainsi, dans les années 1970, se développe au Saguenay-Lac-Saint-Jean une société relativement riche, grouillante, dynamique, qui bouillonne de projets et d'activités, qui offre des opportunités d'affaires et de carrières et qui procure des emplois diversifiés et bien payés à des diplômés universitaires et spécialisés dans les entreprise privées et les institutions publiques.

Mais le ressort casse dès le début des années 1980... L'élan est stoppé, le développement stagne et la région amorce une décroissance, d'abord mineure et ensuite de plus en plus perceptible et accentuée jusqu'à la situation sans précédent que l'on connaît.
Plusieurs facteurs de diverses natures expliquent le phénomène...

- Au début des années 1980, une récession économique mondiale frappe les pays de l'OCDE. Les gouvernements québécois et canadien aboutissent à l'impasse budgétaire et amorcent des politiques de restriction des programmes et de compression des dépenses qui ralentissent et réduisent graduellement et de plus en plus fortement la présence gouvernementale sur le territoire, particulièrement dans les régions.

- Les lendemains du référendum de mai 1980 ont paralysé le Québec dans sa dynamique interventionniste du gouvernement, notamment dans son approche du développement régional.

- La tension politique constante entre Québec et Ottawa continue de nuire à la cohérence et à la cohésion de l'action gouvernementale sur le territoire régional. Une compétition souvent malsaine $s$ 'installe entre les deux paliers de gouvernement.

- Le développement de plus en plus affirmé de l'activité socio-économique canadienne, particulièrement vers 1'Ontario et Toronto et plus généralement vers l'Ouest (Alberta et Colombiebritannique), écarte davantage le Québec de l'axe politique canadien et crée des écarts et un vacuum économique dans le milieu des affaires de Montréal. La région métropolitaine deviendra le principal sujet de préoccupation des politiques de développement du gouvernement québécois et de l'aile politique québécoise au fédéral. L'interventionnisme gouvernemental en région est dès lors évacué des priorités. Les stratégies d'intervention et les principales ressources sont canalisées vers la région montréalaise dans des créneaux de pointe de plus en plus éloignés du tissu régional (avionnerie, industrie pharmaceutique, multimédia, etc.).

- Les années 1980 et 1990 voient se perpétuer tout en s'accélérant un mouvement de contrôle de l'économie régionale par des forces externes qui sapent le développement local (les entreprises, les ressources économiques, les instruments financiers, les infrastructures commerciales et de services, et conséquemment les profits). 
- Le débat constitutionnel (avant et après Meech) relègue dans l'ombre la question régionale au Québec, particulièrement la décroissance des régions périphériques. Les gouvernements transforment graduellement leur politique interventionniste en un discours d'appui à la prise en charge locale et d'aide à la diversification économique des régions. Les sommets économiques de 1984 et de 1995 sont des illustrations éloquentes de la progression de plus en plus affirmée d'une volonté de désengagement de l'État.

- Les effets combinés de la mondialisation des marchés, du développement technologique et de l'actionnariat du capital ont réduit de façon constante et déterminante l'impact économique régional de la présence des grandes entreprises exploitant les ressources hydroélectriques et la forêt.

- Un sentiment d'abandon et d'impuissance perturbe la représentation collective du territoire. Ce sentiment a été accentué et dramatisé par la perception négative des négociations que mène le gouvernement sur l'Approche commune de la part de certains groupes.

\section{Quelques éléments de prospective pour une renaissance régionale}

$\mathrm{Au}$ début $\mathrm{du} \mathrm{XXI}^{\mathrm{e}}$ siècle, la population régionale est confrontée à une forte pression de décroissance de son territoire. La structure économique, basée sur l'exploitation des ressources naturelles en milieu nordique éloigné des marchés, est perçue comme une contrainte majeure par rapport aux nouvelles normes mondiales du développement.

Un puissant sentiment d'urgence pousse les forces vives régionales à réagir, à remettre en question les pratiques établies, à chercher des voies d'avenir, à renouveler la vision de développement du territoire. Il est de plus en plus évident que l'approche traditionnelle de développement ne rencontre plus les intérêts supérieurs de la région, car elle crée désormais du sous-développement local. Le développement économique, tel qu'il est pratiqué dans un contexte de mondialisation, exerce une pression telle sur le Saguenay-Lac-Saint-Jean et sur les régions du moyen nord du Québec qu'il risque de provoquer le déclin du territoire, l'affaiblissement des organisations et l'appauvrissement de ses populations.

Selon les prévisions actuelles, la décroissance démographique et économique régionale est une tendance qui devrait s'accentuer inexorablement dans les prochaines années. À première vue, deux scénarios se présentent aux intervenants :

- gérer au mieux cette tendance jusqu'à ce que soit atteint un plancher qui reflète le nouveau potentiel régional;

- mobiliser d'énormes ressources pour la stopper ou la ralentir significativement en se basant sur les indicateurs économiques traditionnels.

\section{Il est de plus en plus évident que I'approche traditionnelle de développement ne rencontre plus les intérêts supérieurs de la région, car elle crée désormais du sous-développement local.}

Le premier scénario nous paraît inacceptable compte tenu des ressources abondantes et diversifiées de la société régionale, alors que le deuxième est irréaliste si l'on tient compte du poids démographique et politique du Saguenay-Lac-Saint-Jean dans l'ensemble politique québécois et canadien.

Une combinaison judicieuse et dosée des deux scénarios paraît plus appropriée : gérer ouvertement et graduellement la décroissance dans les domaines où elle est irrémédiable tout en intervenant là où une perspective de stabilisation est prévisible et une lueur de renaissance est détectable.

Tout le défi réside évidemment dans la capacité des régions, dont le Saguenay-Lac-Saint-Jean, de dégager une vision collective qui permettra d'identifier des stratégies, de cerner des créneaux d'intervention, de mettre en place des outils et des instruments efficaces et adaptés, de mobiliser les populations et d'investir des ressources.

Nous proposons la vision suivante...

Le devenir de la région passe par une démarche organisée, libre et supportée scientifiquement vers la prise 
en charge, dont les conséquences vérifiables seront une humanisation croissante et créatrice des activités sur le territoire, une évolution continue de la qualité de vie de ses habitants et un accroissement de la richesse collective.

Pour la mettre en œuvre, les acteurs régionaux ne pourront se passer de la prospective territoriale, qui est à la fois une expertise et une démarche sans lesquelles toute intervention est inefficace et démobilisatrice.

La prospective territoriale implique en effet deux volets, différents et complémentaires : a) le volet scientifique (d'où l'importance de tenir des comptabilités claires et acceptées de tous sur les économies de chacune des régions du Québec) et professionnel, qui analyse les grandes tendances de la société et son environnement; b) le volet territorial et collectif, qui est une démarche d'appropriation d'une vision collective élaborée et partagée par les citoyens.

La prospective territoriale renouvellerait notre vision régionale à travers l'approche systémique, celle qui permet de discerner les changements fondamentaux, les transformations profondes, les mutations, la métamorphose. Toutefois, en plus des critères traditionnels plus objectifs, de nouveaux critères devraient enrichir l'évaluation des éléments de renouveau et des facteurs de renaissance inscrits dans la dynamique du territoire.

Ces nouveaux critères sont d'ordre subjectif et concernent la conscientisation et l'humanisation du processus de renaissance, l'élargissement de la conscience collective et de l'autonomie des populations ainsi que la croissance de la qualité de vie, c'est-àdire du capital territorial, social, humain, culturel et naturel.

Toute renaissance devrait être le résultat d'une mobilisation de la population à travers la société civile, de l'émergence de la créativité, de la convergence des capacités et de la mise à contribution des ressources de la région elle-même.

Par société civile, nous entendons tous les acteurs régionaux, autres que l'État et les élus, qui composent et animent la vie régionale à travers les institutions,

les corporations, les groupes et les mouvements populaires. Nous parlons donc des intervenants des milieux économiques, sociaux, culturels, syndicaux, communautaires, environnementaux, sportifs, touristiques, etc.

La collectivité devrait se doter des capacités de vision et des outils de communication stratégiques indispensables à la cohérence et à la cohésion de toute mobilisation qui chercherait à orienter le navire régional sur la voie d'un développement renouvelé et durable. Parce que cette mobilisation doit émerger de la collectivité elle-même, toute initiative pour la mettre en œuvre doit provenir des acteurs de la société civile qui, avec la collaboration active des élus et de l'État (fonds de développement, politiques de décentralisation, etc.), y investissent leur compétence, leur savoir, leur sagesse. À cet égard, les peuples autochtones (Cris, Inuit et Innus) contribuent par leur action à améliorer nos visions de développement de ces territoires où ils sont les premiers occupants depuis des millénaires.

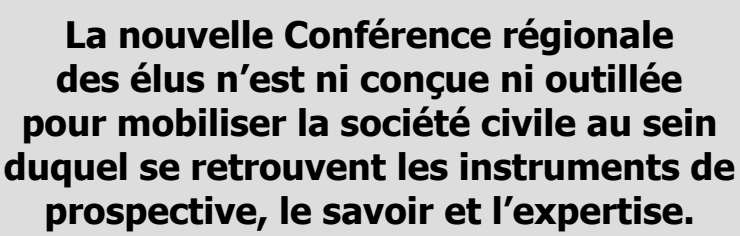

Pour l'heure, on pourrait appeler cet outil de mobilisation de diverses façons: Observatoire de la société civile, Réseau régional de vigie, Centre régional de prospective, Chaire sur les peuples autochtones, etc. L'essentiel est de bien voir qu'il ne s'agit pas d'une autre structure parallèle à celles qui existent, mais que c'est la société civile elle-même qui se constitue en réseau de communication et d'information socialement structuré avec comme objectifs de favoriser et de canaliser l'intelligence collective afin de répondre aux grands défis de l'avenir du territoire.

La nouvelle Conférence régionale des élus n'est ni conçue ni outillée pour mobiliser la société civile au sein duquel se retrouvent les instruments de prospective, le savoir et l'expertise. Sans société civile, le navire régional risque de s'échouer sur les récifs des divisions politiques dans le brouillard de notre ignorance collective. 
Par contre, un réseau incarné dans la société civile dégagerait un espace public où se tisseraient des liens potentiellement féconds avec les élus, l'État et les grandes corporations économiques, l'objectif commun étant de rendre plus efficace la gouvernance régionale tout en induisant la valeur ajoutée de l'information et de la communication aux ingrédients plus traditionnels de la démocratie que sont la représentation et la responsabilité.

\section{Notes}

1 Camil Girard est responsable du Groupe de recherche en histoire de l'Université du Québec à Chicoutimi (GRHUQAC) et professeur associé de l'Institut national de la recherche scientifique, Culture et Société.

2 Laval Gagnon, diplômé en histoire de l'Université du Québec à Chicoutimi, est le coordonnateur régional de Télé-Québec. 


\section{Publicité}

Maîtrise en gestion de projet 